\title{
Butylated hydroxyanisole affects immunomodulation and promotes macrophage phagocytosis in normal BALB/c mice
}

\author{
FANG-MING HUNG ${ }^{1}$, YING-YING CHUANG ${ }^{2,3}$, CHING-SUNG LEE ${ }^{4}$, YUNG-LIANG CHEN $^{3}$, \\ JAI-SING YANG ${ }^{5}$, JEN-JYH LIN ${ }^{6,7}$, KUNG-WEN LU $^{8}$, HUI-YING HUANG ${ }^{9}$, \\ CHIEN-CHIH YU ${ }^{10}$, HSU-FENG LU ${ }^{2,4^{*}}$ and JING-GUNG CHUNG ${ }^{11,12^{*}}$
}

\author{
${ }^{1}$ Department of Surgical Intensive Care Unit, Far Eastern Memorial Hospital, New Taipei 220; \\ ${ }^{2}$ Department of Clinical Pathology, Cheng Hsin General Hospital, Taipei 112; ${ }^{3}$ Department of Medical Laboratory Science \\ and Biotechnology, Yuanpei University, Hsinchu 300; ${ }^{4}$ Department of Restaurant, Hotel and Institutional Management, \\ Fu-Jen Catholic University, New Taipei 242; ${ }^{5}$ Department of Pharmacology; ${ }^{6}$ Graduate Institute of Chinese Medicine; \\ ${ }^{7}$ Division of Cardiology; ${ }^{8}$ School of Post-Baccalaureate Chinese Medicine; ${ }^{9}$ Department of Nutrition; \\ ${ }^{10}$ School of Pharmacy; ${ }^{11}$ Department of Biological Science and Technology, China Medical University, \\ Taichung 404; ${ }^{12}$ Department of Biotechnology, Asia University, Taichung 413, Taiwan, R.O.C.
}

Received September 14, 2011; Accepted December 9, 2011

DOI: $10.3892 / \mathrm{mmr} .2011 .704$

\begin{abstract}
Butylated hydroxyanisole (BHA), a synthetic antioxidant, has been used in fat and fatty foods to prevent oxidative deterioration. However, the functions of BHA on immune responses in normal mice remain elusive. The aim of the present study was to investigate the effects of oral treatment of BHA on immune responses in normal mice in vivo. $\mathrm{BALB} / \mathrm{c}$ mice received various treatments. Blood samples were collected and analyzed. Flow cytometry was used to determine the levels of the cell markers. Results showed that BHA did not significantly affect the weight of the animal body and spleen in normal mice. BHA promoted macrophage phagocytosis from peripheral blood mononuclear cells, but did not alter this process in the peritoneal cavity. Furthermore, BHA did not influence natural-killer cell cytotoxicity in normal mice. Notably, BHA promoted the levels of CD3 (T cells) and decreased the level of CD19 (B cells), but did not significantly affect the levels of $\mathrm{CD} 11 \mathrm{~b}$ (monocytes) and macrophages (Mac-3) in normal mice. Based on these observations it can be concluded that BHA promotes immune responses by increasing $\mathrm{T}$ cells and activating phagocytosis by macrophages in normal mice. However, the molecular mechanisms require further investigation.
\end{abstract}

Correspondence to: Dr Jing-Gung Chung, Department of Biological Science and Technology, China Medical University, No. 91 HsuehShih Road, Taichung 40402, Taiwan, R.O.C.

E-mail: jgchung@mail.cmu.edu.tw

Dr Hsu-Feng Lu, Department of Clinical Pathology, Cheng Hsin General Hospital, No. 45, Cheng Hsin St., Taipei 112, Taiwan, R.O.C. E-mail:ch1835@chgh.org.tw

${ }^{*}$ Contributed equally

Key words: butylated hydroxyanisole, macrophages, natural killer cells, phagocytosis

\section{Introduction}

Leukemia is one of the leading causes of death in humans, and numerous efforts towards the development of adjuvant and neoadjuvant therapies have been made to improve survival rate (1). Leukemia is generally poorly responsive to conventional treatment modalities, such as chemotherapy and radiation therapy (2). Chemotherapeutic agents may induce toxicity and inherent resistance, but there is no consensus regarding optimal therapeutic agents in leukemia. Thus, the development of novel approaches to prevent and treat leukemia is essential.

Butylated hydroxyanisole [BHA; 2(3)-tert-butyl4-hydroxyanisole] is a synthetic phenolic compound, which is widely used as an antioxidant for food products, pharmaceuticals and cosmetics $(3,4)$. BHA has been classified as a $2 \mathrm{~B}$ compound and a possible carcinogen to humans based on the International Agency for Research on Cancer (5). BHA has been reported to be a forestomach carcinogen in rats, mice and hamsters $(6,7)$. However, findings of other reports have demonstrated that BHA shows no evidence of genotoxic activity $(8,9)$ and it prevents chemically-induced tumorigenesis in certain animal tissues $(10,11)$.

Although many studies described the effects of BHA in cancer cell lines in vitro, there is no available information regarding whether BHA affects immune responses of normal mice. Thus, the present study investigated the BHA effect on immune responses in normal mice in vivo. We found that BHA promotes the T-cell level and macrophage phagocytosis in vivo.

\section{Materials and methods}

Materials and reagents. BHA, dimethyl sulfoxide (DMSO), propidium iodide (PI) and trypan blue were obtained from Sigma-Aldrich Corp. (St. Louis, MO, USA). Pharm Lyse ${ }^{\mathrm{TM}}$ lysing buffer was purchased from BD Biosciences (San Jose, 
CA, USA). FITC anti-mouse CD3, PE anti-mouse CD19, PE anti-mouse Mac-3 and FITC anti-mouse CD11b antibodies were purchased from BD Pharmingen Inc. (San Diego, CA, USA). The PHAGOTEST kit was obtained from Glycotope Biotechnology GmbH (Heidelberg, Germany). RPMI-1640, fetal bovine serum (FBS), penicillin-streptomycin and L-glutamine were obtained from Invitrogen Life Technologies (Carlsbad, CA, USA).

Male BALB/c mice. Forty male BALB/c mice, 8 weeks of age and 22-28 $\mathrm{g}$ in weight were obtained from the National Laboratory Animal Center (Taipei, Taiwan, R.O.C.)

BHA treatment. BALB/c mice were randomly divided into four groups to receive different treatments. Group I served as a control $(n=10)$. Group II mice were treated with olive oil as vehicle $(n=10)$. Group III mice were treated with BHA $(100 \mathrm{mg} / \mathrm{kg})$ in olive oil $(\mathrm{n}=10)$. Group IV animals were treated with BHA $(200 \mathrm{mg} / \mathrm{kg})$ in olive oil $(\mathrm{n}=10)$. BHA was administered by oral gavage to the treatment groups daily for up to 3 weeks before being weighed (12).

Body and spleen tissue weights. At the end of treatment, the animals were weighed and blood was withdrawn. Spleen samples were isolated and weighed individually (12).

Immunofluorescence staining for surface markers from normal mice after exposure to BHA. At the end of the treatment, blood samples from the animals were collected and analyzed and individually exposed to $1 \mathrm{X}$ Pharm Lyse ${ }^{\mathrm{TM}}$ lysing buffer (BD Pharmingen Inc.) for lysing of the red blood cells. The samples were then centrifuged for $15 \mathrm{~min}$ at $1,500 \mathrm{rpm}$ at $4^{\circ} \mathrm{C}$. The isolated white blood cells from each animal were stained using FITC anti-mouse CD3, PE anti-mouse CD19, PE anti-mouse Mac-3 and FITC anti-mouse CD11b antibodies before being analyzed to determine the levels of the cell markers using flow cytometry (FACS Calibur ${ }^{\mathrm{TM}}$; Becton-Dickinson, NJ, USA) as previously described $(13,14)$.

Quantification of phagocytic activity of macrophages. Macrophage phagocytosis used the PHAGOTEST kit, as previously described (15). At the end of the treatment, a total of $1 \times 10^{5}$ leukocytes from peripheral blood mononuclear cells (PBMCs) or peritoneal cavity in $100 \mu \mathrm{l}$ whole blood from individual animals of each group were incubated for $1 \mathrm{~h}$ at $37^{\circ} \mathrm{C}$ with fluoresce in isothiocyanate-labelled $E$. coli $(20 \mu \mathrm{l})$. The quenching solution $(100 \mu \mathrm{l})$ was added to the reaction according to the manufacturer's instructions. After the completion of phagocytosis by monocytes/macrophages, DNA was stained according to the manufacturer's protocol. Cells from each animal were analyzed by flow cytometry, as previously described (16). Fluorescence data were collected on 10,000 cells and analyzed using the BD CellQuest software $(15,16)$.

Quantification of natural-killer (NK) cell cytotoxicity. A total of $1 \times 10^{5}$ leukocytes from the spleens of BHA-treated animals in $1 \mathrm{ml}$ of RPMI-1640 were individually cultured in each well of 24-well culture plates for $24 \mathrm{~h}$. Then, $2.5 \times 10^{7}$ of YAC-1 cells were cultured in 15-ml tubes with serum-free RPMI-1640 medium. PKH-67/Dilunt C buffer (Sigma-Aldrich Corp.) was added to the cells, mixed thoroughly for $2 \mathrm{~min}$ at $25^{\circ} \mathrm{C}$, then
$2 \mathrm{ml}$ PBS was added for $1 \mathrm{~min}$. Finally, $4 \mathrm{ml}$ of RPMI-1640 was added for a 10-min incubation, followed by centrifugation at $1,200 \mathrm{rpm}$ at $25^{\circ} \mathrm{C}$. At the same time, the YAC- 1 cells in $100 \mu \mathrm{l}$ were placed on 96-well plates before the addition of the leukocytes from each treatment to the wells for $6 \mathrm{~h}$ and determination of NK cell activity by flow cytometry, as previously described $(13,17)$.

Statistical analysis. The results were shown as the means \pm SD and the difference between the BHA and control groups was analyzed using the Student's t-test. $\mathrm{P}<0.05$ was considered to be significant.

\section{Results}

$B H A$ affected the body and spleen weight in normal BALB/C mice. To investigate whether BHA affects the body and spleen weights of normal mice, body weights of each group were weighed, then spleen tissues were isolated and individually weighed at the end of the BHA treatment. Results shown in Fig. 1 indicate that BHA did not affect the body (Fig. 1A and B) and spleen (Fig. 1C and D) weights of each treatment group, compared to the untreated control group.

BHA affected the surface markers of whole blood cells from normal BALB/c mice. To investigate whether BHA affects the levels of the cell surface markers, leukocytes were isolated from BHA-treated or untreated groups, and then the levels of CD3, CD19, Mac-3 and CD11b were measured. Data are shown in Fig. 2A-D, and indicate that BHA significantly increased the levels of CD3 (100 and $200 \mathrm{mg} / \mathrm{kg} / \mathrm{day}$; 43.57 and 44.89\%; Fig. 2A) and decreased the levels of CD19 (100 and $200 \mathrm{mg} /$ $\mathrm{kg}$ /day; 16.53 and 22.74\%; Fig. 2B), but did not significantly affect the levels of Mac-3 (Fig. 2C) and CD11b (Fig. 2D) when compared to the untreated groups.

BHA promoted phagocytosis by macrophages from PBMC and peritoneal cavity in normal $B A L B / c$ mice. To examine whether BHA affects phagocytosis, the leukocytes from PBMC and the peritoneal cavity of the BHA-treated or oliver oil-treated groups were isolated and phagocytic activity was determined. Results are shown in Fig. 3A and B, indicating that BHA promoted the activity of phagocytosis from PBMC (100 mg/kg/day, 12.86\%; 200 mg/kg/day, 13.72\%; Fig. 3A), but did not significantly affect the activity of phagocytosis from the peritoneal cavity (Fig. 3B).

BHA affected NK cell cytotoxicity of splenocytes from normal $B A L B / c$ mice. The results from NK cell cytotoxicity are shown in Fig. 4, indicating that both target YAC-1 cell ratios of 50:1 and 25:1 were not killed by NK cells from the BHA-treated group when compared to the untreated groups. Moreover, BHA at 100 and $200 \mathrm{mg} / \mathrm{kg} /$ day did not cause any significant difference when compared to the untreated groups (Fig. 4).

\section{Discussion}

In rodents, BHA and its demethylated metabolite tert-butylhydroquinone (tBHQ) act as a potent chemopreventive agent against tumor formation of a variety of carcinogens (14-16,18-21). 
A
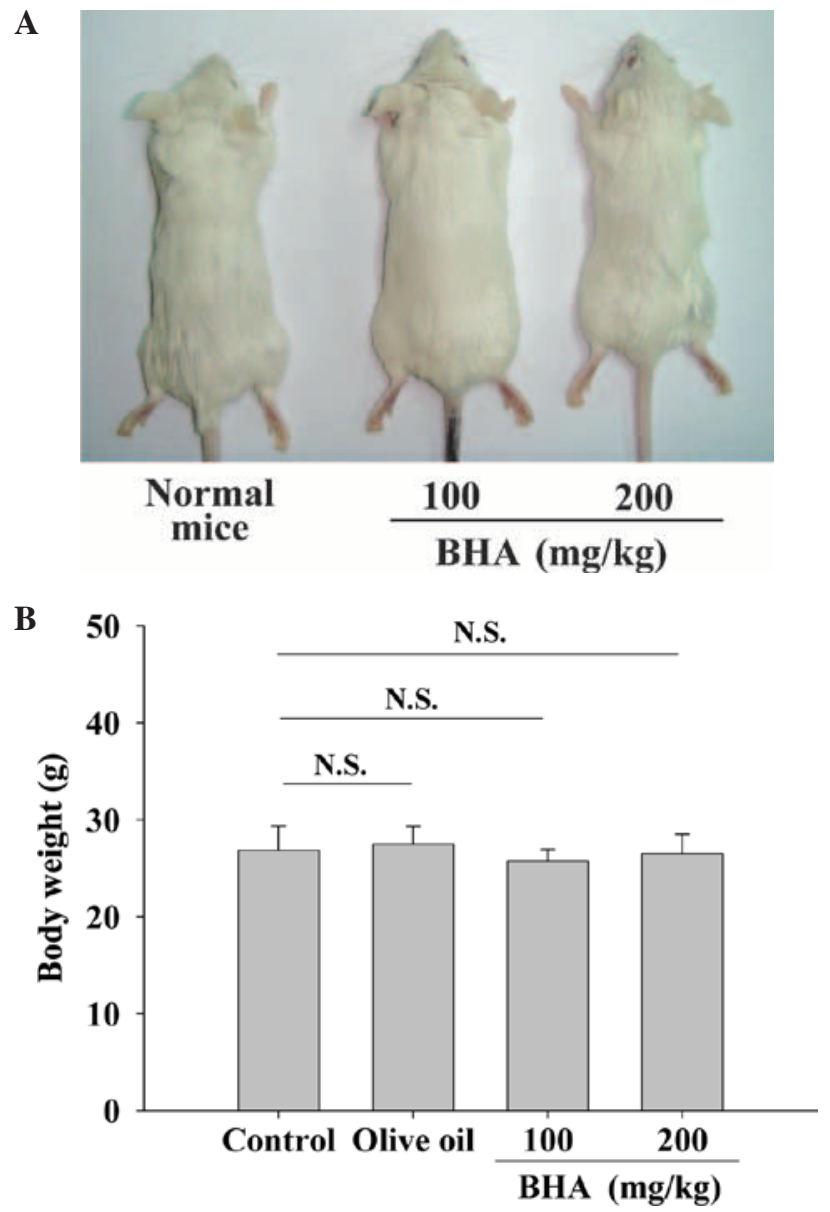

$\mathbf{C}$
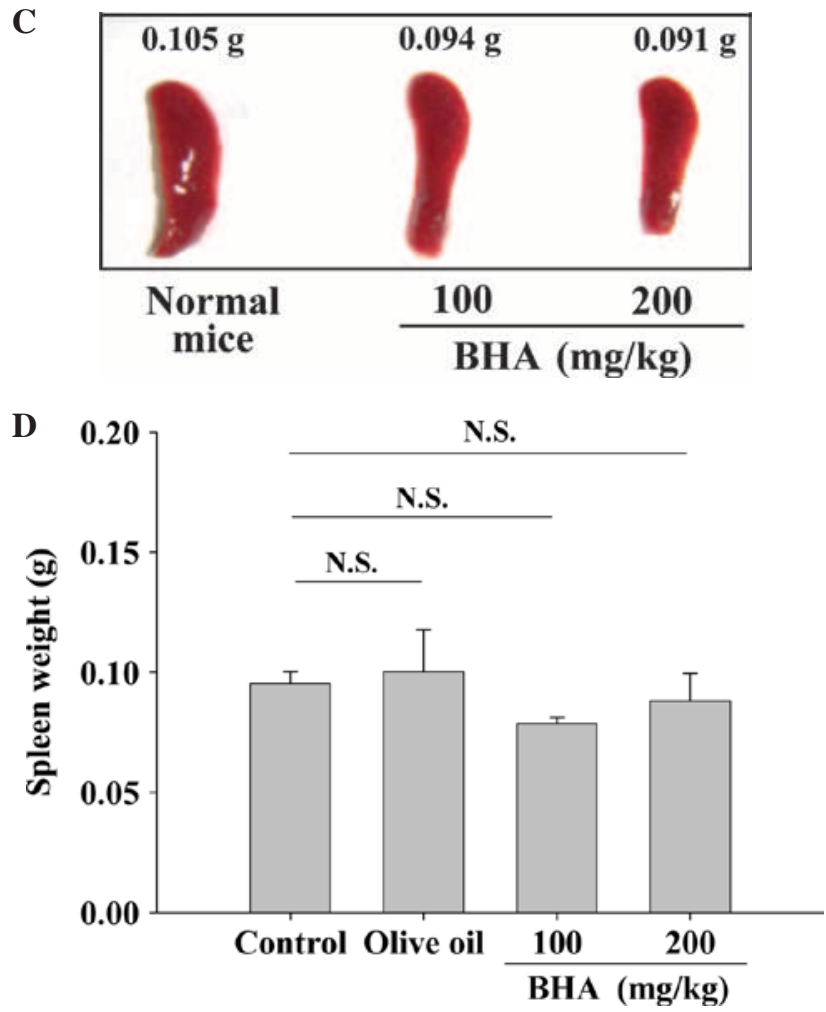

Figure 1. BHA affected the body and spleen weights of normal BALB/c mice. The mice were kept in the animal center for another 3 weeks and then orally treated with or without BHA (100 and $200 \mathrm{mg} / \mathrm{kg} / \mathrm{mouse}$ ) for 3 weeks. The (A) representative animal, (B) body, (C) representative spleen and (D) spleen weights were weighed as described in Materials and methods. Each point is the mean $\pm \mathrm{SD}(\mathrm{n}=10) .{ }^{*} \mathrm{P}<0.05$ was considered significant when compared to the untreated normal mice.
A

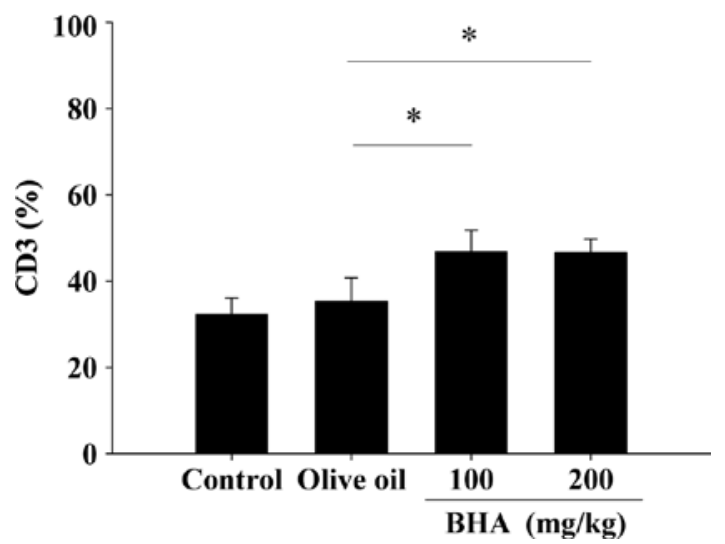

B

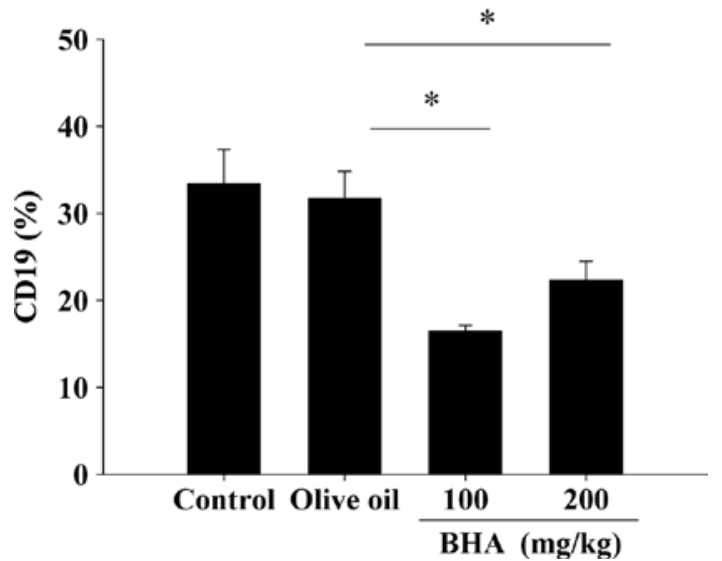

C

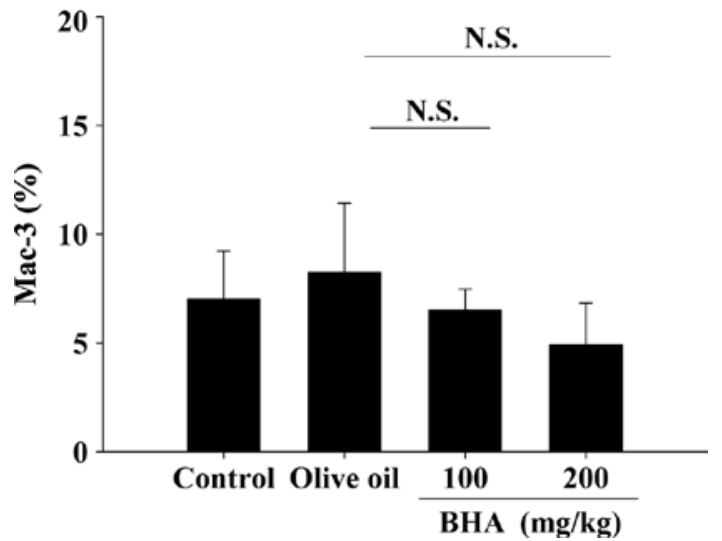

D

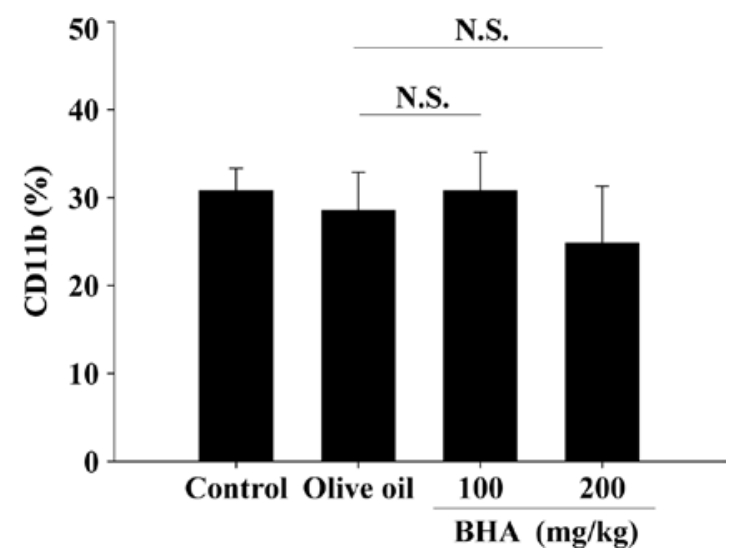

Figure 2. BHA affected the levels of cell markers in white blood cells from normal BALB/c mice at the end of the oral treatment with or without BHA for 3 weeks. Blood was collected from each group and was analyzed for cell markers using flow cytometry as described in Materials and methods: (A) CD3; (B) CD19; (C) Mac-3; (D) CD11b. Data are expressed as the means \pm $\mathrm{SD}$ of three experiments. Each point is the mean $\pm \mathrm{SD}(\mathrm{n}=10){ }^{*} \mathrm{P}<0.05$ was considered significant when compared to the untreated normal mice. 

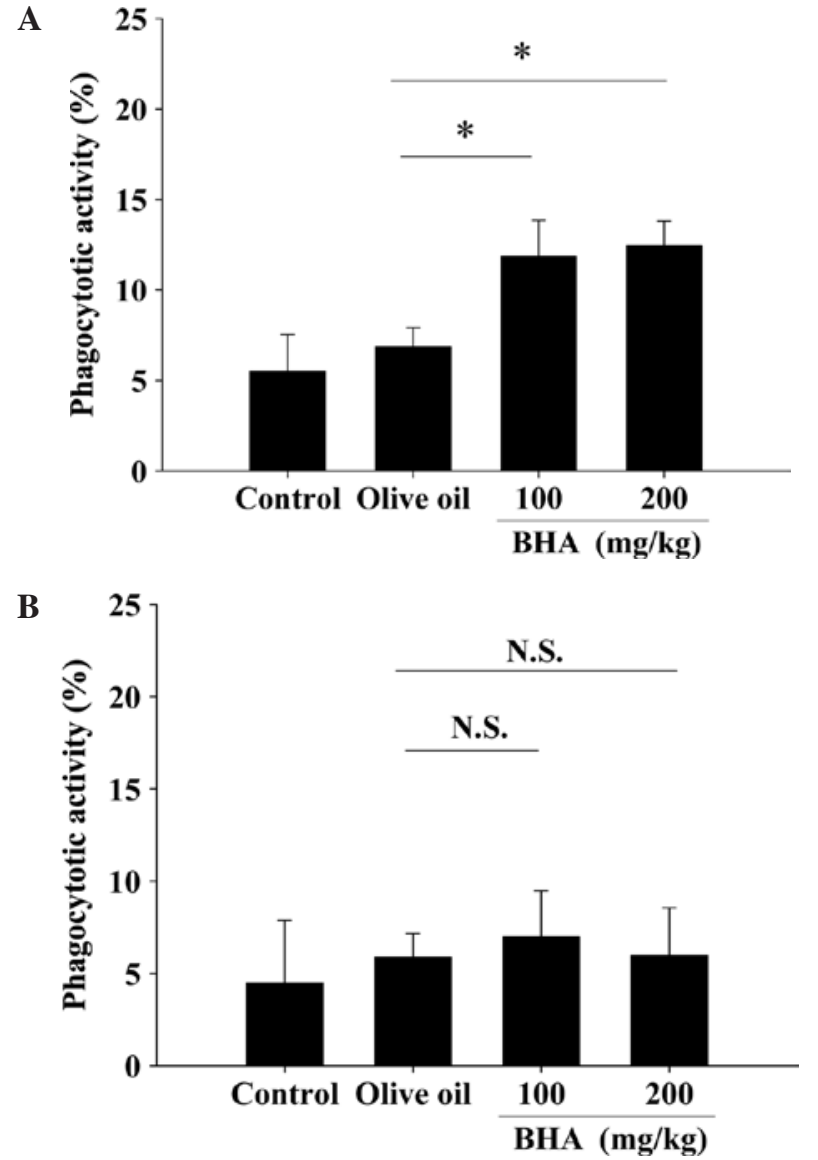

Figure 3. BHA affected the macrophage phagocytosis from normal BALB/c mice. The mice were orally treated with or without BHA for 3 weeks. Macrophages were isolated from (A) PBMC and (B) peritoneal cavity, and the percentages of phagocytosis with phagocyte green fluorescent particles (FITC-E. coli) were determined using flow cytometry, as described in Materials and methods. Each point is the mean $\pm \mathrm{SD}$. ${ }^{*} \mathrm{P}<0.05$ was considered significant when compared to the untreated normal mice $(n=10)$.

BHA is also a potent inducer of phase II detoxifying enzymes, such as glutathione S-transferase (GST) and quinine reductase (QR) (22-24). Although various studies have demonstrated the protective effects of antioxidant BHA on carcinogen-induced carcinogenesis in animal model, these findings are also controversial. In particular, the protective effect was established to determine the influence of certain carcinogens acting upon tissues of given strains of animals. however, the carcinogens that were examined did not protect other tissues of different strains of animals against other types of carcinogens (4). There is currently no available information showing that BHA affects immune responses in normal mice in vivo. Thus, in the present study we investigated the oral administration of BHA, and then examined the immune responses of normal mice in vivo.

Results from flow cytometric assay for cell markers from normal mice after oral treatment of BHA indicated that the percentages of $\mathrm{CD} 3$ (T cells) were significantly increased and the levels of CD19 (B cells) were significantly decreased, but there was no significant difference in the levels of CD11b (monocytes) and Mac-3 (macrophages) (Fig. 2A-D). Furthermore, there was no effect on the body, liver (data not shown) or spleen weight of normal mice after oral treatment with BHA (Fig. 1A-C).

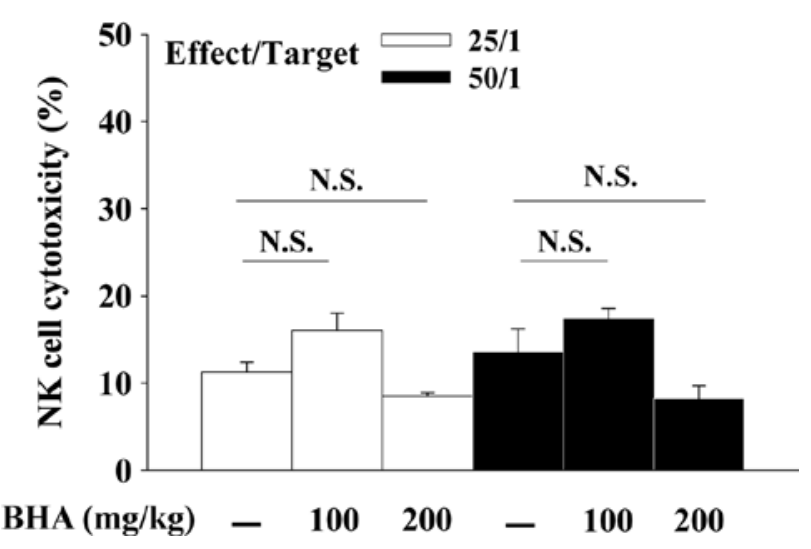

Figure 4. BHA affected the NK cell cytotoxicity from normal BALB/c mice The mice were orally treated with or without BHA for 3 weeks. The YAC-1 target cells were killed by NK cells of splenocytes from the mice after treatment with or without BHA by oral administration at 100 and $200 \mathrm{mg} / \mathrm{kg} / \mathrm{day}$ in target cell ratios of 25:1 and 50:1, as described in Materials and methods. Each point is the mean $\pm \mathrm{SD}$. " $\mathrm{P}<0.05$ was considered significant when compared to the WEHI-3 leukemia mice $(\mathrm{n}=10)$.

We also found that BHA (100 and $200 \mathrm{mg} / \mathrm{kg}$ ) promoted the phagocytosis of macrophages from PBMC (Fig. 3A) rather than that from peritoneal cavity (Fig. 3B). Both macrophage phagocytosis and NK cell cytotoxicity are involved in immune responses after animals are exposed to antigen (14-16). However, BHA $(100$ and $200 \mathrm{mg} / \mathrm{kg}$ ) treatment did not alter the cytotoxicity of NK cells (Fig. 4). It has been reported that the estimated no-effect-level of BHA in food is approximately $250 \mathrm{mg} / \mathrm{kg}$ body weight per day (4).

BHA may fully exert its chemopreventive action by rendering the toxic free radicals, enhancing the process of excretion of foreign compounds, inhibiting the metabolic conversion of carcinogens to harmful derivatives, inhibiting the binding of these carcinogenic metabolites to the nuclear DNA and modifying the immune defense mechanisms of the organism (4).

Based on these observations, a conclusive result regarding BHA promoted immune responses could not be reached. Although we observed macrophage phagocytosis in mice after BHA exposure, there was a decrease of NK cell cytotoxicity. Further studies are required to determine whether or not BHA acts as a potent immunological adjuvant in animal models in vivo.

\section{Acknowledgements}

This study was supported by the grant DOH100-TD-C-111-005 from the Taiwan Department of Health, China Medical University Hospital Cancer Research Center of Excellence.

\section{References}

1. Schutz FA and Oh WK: Neoadjuvant and adjuvant therapies in prostate cancer. Urol Clin North Am 37: 97-104, 2010.

2. Belasco JB, Goldwein JW, Simms S, Griffin G, D'Angio G and Lange B: Hypofractionated moderate dose radiation, intrathecal chemotherapy, and repetitive reinduction/reconsolidation systemic therapy for central nervous system relapse of acute lymphoblastic leukemia in children. Med Pediatr Oncol 34: 125-131, 2000.

3. Hocman G: Chemoprevention of cancer: selenium. Int J Biochem 20: 123-132, 1988. 
4. Hocman G: Chemoprevention of cancer: phenolic antioxidants (BHT, BHA). Int J Biochem 20: 639-651, 1988.

5. Hirose M, Masuda A, Tsuda $H$, Uwagawa $S$ and Ito $N$ : Enhancement of BHA-induced proliferative rat forestomach lesion development by simultaneous treatment with other antioxidants. Carcinogenesis 8: 1731-1735, 1987.

6. Ito N, Fukushima S, Hagiwara A, Shibata M and Ogiso T: Carcinogenicity of butylated hydroxyanisole in F344 rats. J Nat Cancer Inst 70: 343-352, 1983.

7. Clayson DB, Iverson F, Nera EA and Lok E: The significance of induced forestomach tumors. Annu Rev Pharmacol Toxicol 30 441-463, 1990

8. Williams GM, McQueen CA and Tong C: Toxicity studies of butylated hydroxyanisole and butylated hydroxytoluene. I. Genetic and cellular effects. Food Chem Toxicol 28: 793-798, 1990.

9. Jos A, Repetto G, Rios JC, et al: Ecotoxicological evaluation of the additive butylated hydroxyanisole using a battery with six model systems and eighteen endpoints. Aquat Toxicol 71: 183-192, 2005.

10. Williams GM, Tanaka T and Maeura Y: Dose-related inhibition of aflatoxin B1 induced hepatocarcinogenesis by the phenolic antioxidants, butylated hydroxyanisole and butylated hydroxytoluene. Carcinogenesis 7: 1043-1050, 1986.

11. Williams GM and Iatropoulos MJ: Inhibition of the hepatocarcinogenicity of aflatoxin B1 in rats by low levels of the phenolic antioxidants butylated hydroxyanisole and butylated hydroxytoluene. Cancer Lett 104: 49-53, 1996.

12. Lin CC, Kuo CL, Lee MH, et al: Extract of Hedyotis diffusa Willd influences murine leukemia WEHI-3 cells in vivo as well as promoting $\mathrm{T}$ - and $\mathrm{B}$-cell proliferation in leukemic mice. In Vivo 25: 633-640, 2011.

13. Tsou MF, Tien N, Lu CC, et al: Phenethyl isothiocyanate promotes immune responses in normal $\mathrm{BALB} / \mathrm{c}$ mice, inhibits murine leukemia WEHI-3 cells, and stimulates immunomodulations in vivo. Environ Toxicol: 27 May, 2011 (E-pub ahead of print).
14. Mulligan JK, Lathers DM and Young MR: Tumors skew endothelial cells to disrupt NK cell, T-cell and macrophage functions. Cancer Immunol Immunother 57: 951-961, 2008.

15. Shan BE, Zhang JY and Li QX: [Human T cell and monocyte modulating activity of Rhizoma typhonii in vitro]. Zhongguo Zhong Xi Yi Jie He Za Zhi 21: 768-772, 2001.

16. Thomas PT, Ratajczak HV, Aranyi C, Gibbons R and Fenters JD: Evaluation of host resistance and immune function in cadmiumexposed mice. Toxicol Appl Pharmacol 80: 446-456, 1985.

17. Chen YL, Lu HF, Hung FM, et al: Danthron inhibits murine WEHI-3 cells in vivo, and enhances macrophage phagocytosis and natural killer cell cytotoxic activity in leukemic mice. In Vivo 25: 393-398, 2011

18. Wattenberg LW: Chemoprevention of cancer. Cancer Res 45: 1-8, 1985.

19. Wattenberg LW: Inhibition of neoplasia by minor dietary constituents. Cancer Res 43: S2448-S2453, 1983.

20. King MM and McCay PB: Modulation of tumor incidence and possible mechanisms of inhibition of mammary carcinogenesis by dietary antioxidants. Cancer Res 43: S2485-S2490, 1983.

21. Barrett JC and Wiseman RW: Cellular and molecular mechanisms of multistep carcinogenesis: relevance to carcinogen risk assessment. Environ Health Perspect 76: 65-70, 1987.

22. Benson AM, Batzinger RP, Ou SY, Bueding E, Cha YN and Talalay P: Elevation of hepatic glutathione $S$-transferase activities and protection against mutagenic metabolites of benzo(a) pyrene by dietary antioxidants. Cancer Res 38: 4486-4495, 1978.

23. Sparnins VL, Chuan J and Wattenberg LW: Enhancement of glutathione S-transferase activity of the esophagus by phenols, lactones, and benzyl isothiocyanate. Cancer Res 42: 1205-1207, 1982.

24. Sparnins VL, Venegas PL and Wattenberg LW: Glutathione S-transferase activity: enhancement by compounds inhibiting chemical carcinogenesis and by dietary constituents. J Natl Cancer Inst 68: 493-496, 1982 